Urologe 2011 · 50:543-544

DOI 10.1007/s00120-011-2524-8

Online publiziert: 23. April 2011

(c) Springer-Verlag 2011

\author{
M. Westenfelder \\ Sektion Kinderurologie und plastisch-rekonstruktive Urologie, \\ HELIOS-Klinikum Krefeld
}

\title{
Aktuelle Themen aus der Kinderurologie
}

Die Entwicklung der Kinderurologie ging in Deutschland langsamer vonstatten als z. B. in England, USA und Frankreich. Regional sehr unterschiedlich wurden dann aber in den letzten Jahrzehnten sehr große Fortschritte erreicht, die dem internationalen Standard entsprechen. Davon zeugen die folgenden Arbeiten.

Als operatives Fach mit z. T. auch sehr großen Operationen von Kindern sind wir auf eine erfahrene Kinderanästhesie und ein gutes postoperatives Schmerzmanagement angewiesen. Dafür war die Kaudalanästhesie schon ein großer Fortschritt. Für größere und längere Eingriffe ist sie aber nicht ausreichend. Hierfür hat sich in den letzten 15 Jahren die Periduralanästhesie bei Kindern sehr bewährt. Zentren, die große Eingriffe durchführen, sollten nicht ohne diese Narkoseform auskommen müssen. Dies bedeutet, dass sich unsere Anästhesisten mit dieser Technik vertraut machen müssen und wir sie routinemäßig verwenden. Wie und mit welchen Vorteilen zeigt sehr anschaulich die gemeinsame Arbeit von Kinderurologie und Anästhesie von P. Rubenwolf, B. Koller, I. Rübben, A. K. Ebert, F. Pohl und W. H. Rösch aus Regensburg mit dem Titel „Perioperatives Schmerzmanagement bei großen, plastisch rekonstruktiven Eingriffen in der Kinderurologie - ein Plädoyer für die Periduralanästhesie“.

Harnableitungen sind weiterhin wichtige Maßnahmen, um bei Blasenverlust oder Funktionsausfall die Lebensqualität der Kinder zu erhalten oder deutlich zu verbessern. Früher wurden sie, v. a. bei Spina bifida und neurogenen Blasen, sehr häufig, bis viel zu häufig ausgeführt. Ein besseres Verständnis der Pathologie und die starke Abnahme der schweren Fälle mit Spina bifida macht die Harnableitung auch heute noch notwendig, aber vergleichsweise doch sehr viel seltener. Vor allem in der Zeit der 196oer bis Anfang der 1980er Jahre wurden Harnableitungen in den USA sehr häufig auch bei funktionellen Blasenentleerungsstörungen angelegt. Mit zunehmendem Verständnis der Pathophysiologie dieser Blasenentleerungsstörungen und durch die grundlegenden Arbeiten von J. Lapides [1] wurden dann kaum noch Harnableitungen bei diesen Kindern durchgeführt. Die Ära der Undiversions begann. In Deutschland hatte dies erfreulicherweise keine so große Rolle gespielt. Bei Kindern mit langer Lebenserwartung hat die Wahl des Harnableitungsverfahrens ungleich größere Bedeutung als bei Erwachsenen. Langzeitkomplikationen metabolischer und onkologischer Art sind von viel größerer Bedeutung als bei Erwachsenen, und die Sicherheit der Technik ist zusätzlich entscheidend. Die Übersichtsarbeit von R. Stein, A. Schröder, J. W. Thüroff aus Mainz mit dem Titel „Harnableitung bei Kindern. Spezielle Berücksichtigung der Langzeitkomplikationen " liefert dazu eine hervorragende Zusammenfassung, und jeder, der Harnableitungen bei Kindern anlegt, sollte diese Arbeit sorgfältig lesen, um seinen Wissensstand zu aktualisieren.

In Deutschland brauchte (und braucht es noch) sehr lange, bis sich die Erkenntnis zur Pathophysiologie und klinischen Bedeutung von funktionellen Blasenentleerungsstörungen durchsetzte. Dass die Meatusstenose beim weiblichen Geschlecht oder der Moormann-Ring eher
Phantome als Realität waren, scheint weitgehend aber keinesfalls flächendeckend akzeptiert. Verursacht werden diese $\mathrm{Zu}$ stände durch ein Spektrum an funktionellen Blasenentleerungsstörungen, deren Ursprung in der Komplexizität unserer Blaseninnervation mit kompletter Umstellung ihrer Funktion beim Neugeborenen und Säugling zur Funktion im Kindes- und Erwachsenenalter begründet ist. Dabei wird die unwillkürliche Steuerung durch die willkürliche Steuerung abgelöst. Dies bereitet in der Umstellungsphase häufige, aber in der Initialphase gut behandelbare Probleme. Darauf geht die Arbeit „Nicht neurogene Blasenfunktionsstörungen und vesikoureteraler Reflux bei Kindern“von I. Rübben, M. Goepel und J.D. van Gool aus Essen/Velbert ein, insbesondere auf die Auswirkung bei gleichzeitig bestehendem VUR.

Große und echte Fortschritte hat unser heutiges Verständnis der Pathologie der Anomalien des oberen Harntraktes gebracht. Nicht nur, dass wir quasi in jedem Alter diese Anomalien mit den entsprechenden Techniken korrigieren können, sondern vielmehr, dass wir heute wissen, wann wir sie nicht operieren müssen und wann für eine Operation der beste Zeitpunkt ist. Dazu liefern S. Rogenhofer, S.C. Müller aus Bonn einen sehr übersichtlichen und präzisen Beitrag mit dem Titel „Harntransportstörungen (Hydronephrosen). Dringlichkeiten der Interventionen und empfohlene Zeitpunkte der Korrekturen“, der ebenfalls zur Pflichtlektüre aller gehören sollte, v. a. aber für diejenigen, die noch immer davon überzeugt sind, durch „heroische Früheingriffe" Kindern etwas Gutes zu tun. Die damit 
immer wieder eintretenden Komplikationen lassen sich durch nichts rechtfertigen.

Dies gilt auch für den Maldescensus testis, v. a. der nicht palpablen Hoden. Deren Behandlung hat nichts mit der „einfachen Orchidopexie“ zu tun. Ist der Hoden intraabdominell gelegen und ist die Operation schwierig bis sehr schwierig, sollte sie erfahrenen Ärzten vorbehalten bleiben. Diese werden sich dann durch eine Laparoskopie absichern, um den Eingriff so sicher wie möglich angehen zu können, falls möglich einzeitig (Koff-Verfahren), zweizeitig mit Klippen der Gefäße (Fowler-Stephens) oder nur laparoskopisch und dann idealerweise auch wieder einzeitig. Die Erfahrung besteht dann nicht nur darin, diese Techniken zu beherrschen, sondern auch darin zu wissen, wann welche Technik angewendet wird, um den Hoden zu erhalten - denn weiterhin ist die Rate an Hodenatrophien nach diesen Eingriffen zu hoch. Dazu findet sich in diesem Heft der aufschlussreiche Beitrag „Ergebnisse der laparoskopischen Orchidopexie beim nicht palpablen Hoden" von C. Neissner, A.K. Ebert und W. H. Rösch aus Regensburg.

Kinderurologisches Dauerthema ist nicht nur der VUR, sondern auch die Hypospadie. Dazu finden sich in dieser Ausgabe 2 Beiträge. „Aktuelle Trends in der Behandlung primärer Hypospadien“ werden von M. Riccabona aus Linz vorgestellt. Dieser Beitrag zeigt, dass Hypospadien nicht mit Operationsmethoden korrigiert werden, sondern mit schlüssigen Konzepten und Erfahrung. Hält sich der Operateur nicht daran, entstehen Komplikationen aller Art bis hin zu den schwersten Hypospadiekrüppeln, bei denen das Gewebe zerstört wird und die Psyche des Kindes und der Familie traumatisiert ist. Wie M. Musch und M. Moster in ihrem Beitrag „Operative Trends bei sekundären Hypospadien“ demonstrieren, werden diese sekundären Formen oder verkrüppelte Organe auch mit Techniken und Konzepten, v. a. aber mit Erfahrung korrigiert.

Feminisierungsoperationen bei intersexuellem Genitale stehen Hypospadieoperationen in ihrer Komplexizität in nichts nach. Auch bei diesen wurden, obwohl sie vergleichsweise selten sind, große Fortschritte gemacht. Ganz im Gegensatz zu den Korrekturen anderer Anomalien kam es hier aus sehr verschiedenen Gründen zu der fast absurden Situation, in der sich Betroffene, Selbsthilfeorganisationen, Psychologen, Juristen, Politiker, Medien und Journalisten in die Behandlung einmischten mit der Forderung, ein drittes Geschlecht (das der Hermaphroditen) zu schaffen und keine Operationen mehr zuzulassen - dies auch gegen den Willen der Eltern. Auch Ärzte haben sich dieser Meinung angeschlossen, so dass die Verunsicherung sehr groß wurde. Operationen wurden abgesagt mit Androhung juristischer Konsequenzen, und z. B. in den USA ist kaum noch ein Arzt gewillt, eine derartige Operation durchzuführen. Die Analyse der rechtlichen Situation durch Juristen, die sich überwiegend mit diesen Problemen befassen, zeigt aber, dass in allen zivilisierten Ländern die Gesetze eindeutig festlegen, dass ausschließlich die Eltern es sind, die über die Behandlung ihrer Kinder entscheiden und dass es keinerlei rechtliche Grundlagen gibt, politisch andere Entscheidungswege zu schaffen (s. Beitrag Westenfelder).

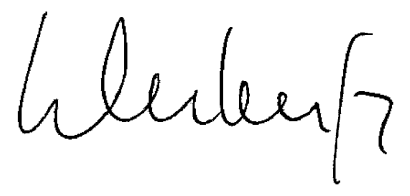

M. Westenfelder

\section{Korrespondenzadresse}

Prof. Dr. M. Westenfelder

Sektion Kinderurologie und

plastisch-rekonstruktive Urologie, HELIOS-Klinikum Krefeld,

Lutherplatz 40, 47805 Krefeld

martin.westenfelder@helios-kliniken.de

\section{Literatur}

1. Lapides J (1979) Mechanisms of urinary tract infection. Urol XIV: 217-225

\section{INVITATION}

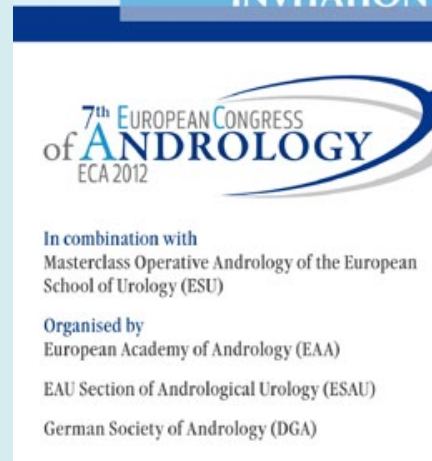

28 November-1 December 2012 Berlin (DE)

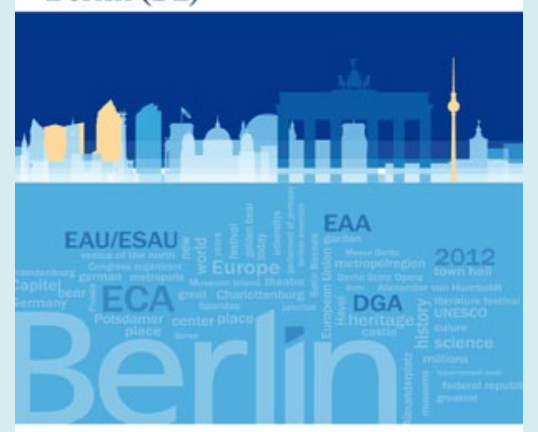

www.andrology2012.de

\section{7th European Congress of Andrology - ECA 2021}

Berlin, 28 November - 1 December 2012

In combination with Masterclass Operative Andrology of the European School of Uro$\log (\mathrm{ESU})$

Organised by European Academy of Andrology (EAA) EAU Section of Andrological Urology (ESAU)

German Society of Andrology (DGA)

Seminaris CampusHotel Berlin

Science \& Conference Center

Takustraße 39

14195 Berlin (Germany)

www.andrology2012.de

www.conventus.de 